\title{
MORE ACCURATE MODELLING OF DIGITAL SUBSCRIBER LINES WITH INHOMOGENEITY
}

The digital subscriber lines are still base of access network to provide Internet connectivity to the public. The transmission capacity is continually increased and performance of digital subscriber lines must be estimated for network provider use. This paper describes two ways for correction of digital subscriber line modelling. The resulting bitrates are calculated with respect to the length of the line and the number of disturbance sources. The article brings new ideas for simulations and calculations of transmission function for line with inhomogeneity and calculations new value of code gain.

Keywords: Access network, model of twisted pair, inhomogeneity, digital subscriber lines.

\section{Introduction}

In May 2011, installation of VDSL2 (Very-high-speed digital subscriber line) was started by Telefonica Czech Republic. The spectral profile 998ADE17 was chosen for VDSL2 lines operating in the access network owned in the Czech Republic [1]. In a year of operation, practical experience with the operation of VDSL is already available and we proceeded to correct our simulation model for digital subscriber lines [2]. The VDSL lines are widely used for the transmission video-streams of IPTV (Internet Protocol Television). The required bandwidth and quality of video stream on higher layers of communication model is researched in many works, for example [3]. This article is focused to transmission directly on the physical medium and the physical layer. Two major problems were identified with precision modelling of digital subscriber lines on the physical layer.

Firstly, transmission function of the line must be accurate. A real telecommunication line can be simplified as a homogeneous line with evenly distributed electrical parameters. As the frequency grows, the inhomogeneities along the line have more influence.

Secondly, code gain of the modem must be updated. The code gain is depended on a vendor and its error correction and equalization algorithms implementation.

Both problems are described and analysed in this article and practices are recommended to address them. Another big problem of metallic lines is crosstalk, which was addressed in many articles, such as [4] and [5].

\section{Types inhomogeneities}

The telecommunications practice uses the following classification for specific types of inhomogeneities that can appear in subscriber lines in local access networks; they have originated from the analogy technology:

- Inhomogeneities of type 1 - they include transitions between different core diameters, transitions between different materials (copper-aluminium), and transitions between different cable arrangements (pairs-quads).

- Inhomogeneities of type 2 - representing "broken" quads, pairs, pair with bad insulation, interruptions.

- Inhomogeneities of type 3 - they originate from disobedience of rules for connecting groups (bundles) and layers (positions).

This classification is, however, insufficient for digital subscriber lines that occupy the frequency bands up to tens of $\mathrm{MHz}$, and therefore it is desirable to introduce the classification according to Table 1. The authors have further divided the individual types into subtypes that are identified by lower-case letters.

It is particularly important to introduce the inhomogeneities of Type 0 that are characterized by inhomogeneity of the cable section itself resulting from imperfect manufacturing and installing, and also from unmatched characteristic impedances of terminal devices and the line section in the operating frequency range (compromise termination) due to extremely wide band.

Type 1 inhomogeneities are caused by the branched access network topology and they must be taken into account in real oper-

\footnotetext{
* Jiri Vodrazka, Petr Jares, Boris Simak

Department of Telecommunication Engineering, Faculty of Electrical Engineering, Czech Technical University in Prague, Czech Republic,

E-mail: vodrazka@feld.cvut.cz
} 
ation. Type 2 inhomogeneities, on the other hand, have the nature of impedance faults, and therefore they should be eliminated by manufacturing and installing; if they occur, the affected cable elements cannot be used for transmission. Type 3 inhomogeneities do not introduce more serious problem than type $0 \mathrm{a}$ and their consequences include mainly crosstalk. It is not possible to state that these consequences are just negative, since a change of layer causes changes in disturbance nature along the line, which may influence the even crosstalk conditions inside the cable tree in a positive way. Type 4 has been also introduced that includes parasite open-ended (4a) as well as functional (4b) taps on a line. The open-ended taps should be eliminated (if possible), while the functional taps should be correctly terminated. Type 5 is linked to additional elements in the access network, such as patch panels, distribution frames, splitters, etc.

Types of inhomogeneities

Table 1

\begin{tabular}{|c|c|c|}
\hline Type & Description & Consequences \\
\hline 0 & \multicolumn{2}{|l|}{ Cable Inhomogeneities } \\
\hline $0 \mathrm{a}$ & $\begin{array}{l}\text { Manufacturing and instal- } \\
\text { lation imperfections }\end{array}$ & $\begin{array}{l}\text { Partial reflections, irregular } \\
\text { ripple of the characteristics }\end{array}$ \\
\hline $0 \mathrm{~b}$ & $\begin{array}{l}\text { Compromise line } \\
\text { termination }\end{array}$ & $\begin{array}{l}\text { Partial reflections, regular ripple } \\
\text { of the characteristics }\end{array}$ \\
\hline 1 & \multicolumn{2}{|l|}{ Line Inhomogeneities } \\
\hline 1a & Cable type change & $\begin{array}{l}\text { Partial reflections, regular ripple } \\
\text { of the characteristics }\end{array}$ \\
\hline $1 b$ & $\begin{array}{l}\text { Cable construction } \\
\text { change }\end{array}$ & \\
\hline 2 & \multicolumn{2}{|l|}{ Impedance defects } \\
\hline $2 \mathrm{a}$ & $\begin{array}{l}\text { Mismatching of cable ele- } \\
\text { ments }\end{array}$ & $\begin{array}{l}\text { Major damage of line function } \\
\text { with rapid growth of attenuation }\end{array}$ \\
\hline $2 b$ & Insulation leakage & \\
\hline $2 \mathrm{c}$ & Conductor interruption & \\
\hline 3 & Position change & $\begin{array}{l}\text { Partial reflections, irregular } \\
\text { ripple of the characteristics }\end{array}$ \\
\hline 4 & \multicolumn{2}{|l|}{ Taps } \\
\hline $4 a$ & Open-ended taps & $\begin{array}{l}\text { Strong reflections and local maxi- } \\
\text { mums of attenuation }\end{array}$ \\
\hline $4 b$ & Functional taps & $\begin{array}{l}\text { Reflections and local maximums } \\
\text { of attenuation }\end{array}$ \\
\hline 5 & \multicolumn{2}{|l|}{ Added components } \\
\hline $5 a$ & $\begin{array}{l}\text { Patch cords and cables } \\
\text { on MDF }\end{array}$ & $\begin{array}{l}\text { Add attenuation and partial } \\
\text { reflections }\end{array}$ \\
\hline $5 b$ & $\begin{array}{l}\text { Distribution frames and } \\
\text { cross-connects }\end{array}$ & $\begin{array}{l}\text { Add attenuation and partial } \\
\text { reflections }\end{array}$ \\
\hline $5 c$ & Splitters & $\begin{array}{l}\text { Impact on parameters for low } \\
\text { frequency }\end{array}$ \\
\hline
\end{tabular}

\section{Model of line with inhomogeneities}

The transmission function of a line is influenced by many factors, and the first option is to model it according to [6] as an environment with multipath propagation:

$$
H(f)=\sum_{i=1}^{N} g_{i} \cdot e^{-\alpha(f) \cdot I_{i}} \cdot e^{-j 2 \pi \cdot f T_{i}}
$$

where $g_{i}$ is the path weight, $T_{i}$ is the delay of a path with length $l_{i}$, $N$ is number of paths and $\alpha$ is specific attenuation.

Another modelling option is to use the telegraph equations, in this case not with constant coefficients, but with variable ones, i.e. primary parameters RLCG depending on the position within the line. Simplified model can be used for practical applications, which is based on real conditions in access networks - the inhomogeneities are not simulated continuously, but discretely, as a limited number of impedance mismatches on the line. In such a case we can model the line as a cascade of individual sections with different parameters and lengths.

If we want to determine the transmission function of a line composed of several sections with different parameters and containing also taps or other elements, we can express it from the product of matrices describing the individual cascaded sections. We should use the cascade parameters of a two-port network ABCD [7]. The resulting matrix of the cascade will be given by the product of the sectional matrices:

$$
A=A_{1} \cdot A_{2} \ldots A_{n}=\left[\begin{array}{ll}
a(f) & b(f) \\
c(f) & d(f)
\end{array}\right]
$$

where $A_{1}$ to $A_{n}$ are matrices describing the cascaded elements and $a(f)$ to $d(f)$ are the resulting parameters of the entire cascade.

Similar procedure can be applied also to modelling of lines with inhomogeneity. The model will be formed by matrices describing homogeneous sections of various lengths. Between each two homogeneous sections there will be inserted inhomogeneities of different nature. The entire cascade will be terminated by a homogeneous section. There are two possibilities of defining the inhomogeneities: either using the impedance defects as combinations of serial and parallel impedances (suitable for less serious defects), or using electrically short sections with different secondary parameters; also, a combination of both methods can be used (suitable for strong inhomogeneities).

Then we can describe a section of a homogeneous line with length $l_{i}$, characteristic impedance $Z_{c i}$ and propagation coefficient $\gamma_{i}$ using the following matrix:

$$
A_{L i}=\left[\begin{array}{cc}
\left(\cosh \left(\gamma_{i}(f) \cdot l_{i}\right)\right) & \left(Z_{C i}(f) \cdot \sinh \left(\gamma_{i}(f) \cdot l_{i}\right)\right) \\
\left(\frac{\sinh \left(\gamma_{i}(f) \cdot l_{i}\right)}{Z_{C i}(f)}\right) & \left(\cosh \left(\gamma_{i}(f) \cdot l_{i}\right)\right)
\end{array}\right]
$$

Impedance $Z_{S}$ that is in series between the input and output contacts will be expressed using the matrix (4); it can be used if 
there is an inhomogeneity caused by increased specific resistance or inductance (e.g. contact resistance in cross-connect and distribution frames).

$$
A_{S}=\left[\begin{array}{cc}
1 & Z_{S} \\
0 & 1
\end{array}\right]
$$

Impedance $Z_{p}$ that is in parallel with the common input and output contacts will be expressed using the matrix (5); it can be used if there is an inhomogeneity caused by increased specific leakage or capacitance (e.g. capacitance of cross-connect and distribution frames).

$$
A_{p}=\left[\begin{array}{cc}
1 & 0 \\
\frac{1}{Z_{p}} & 1
\end{array}\right]
$$

The resulting matrix will be calculated as a product of odd number of partial matrices $(1+2 n)$ as follows:

$$
A=A_{L 1} \cdot \prod_{i=2}^{n}\left(A_{D i} \cdot A_{L i}\right)
$$

where $A_{L i}$ are matrices describing homogeneous sections (Lines) and $A_{D i}$ are matrices modelling the inhomogeneities (Defects), but with different secondary parameters $Z_{c i}$ and $\gamma_{i}$ than in the matrix $A_{L i}$ (3). The number of matrices depends on the required nature of inhomogeneities. For practical use, modelling of inhomogeneity by electrically short sections of lines with different secondary parameters can be recommended. The formula (6) can then be rewritten as a product of matrices, as follows:

$$
A=A\left(\gamma_{1}, Z_{c 1}, l_{L 1}\right) \cdot \prod_{i=2}^{n}\left[A\left(\gamma_{D i}, Z_{c D i}, l_{D i}\right) \cdot A\left(\gamma_{L i}, Z_{c L i}, l_{L i}\right)\right]
$$

where $l_{L i}$ are the homogeneous sections lengths and $l_{D i}$ are the lengths of sections modelling the inhomogeneity.

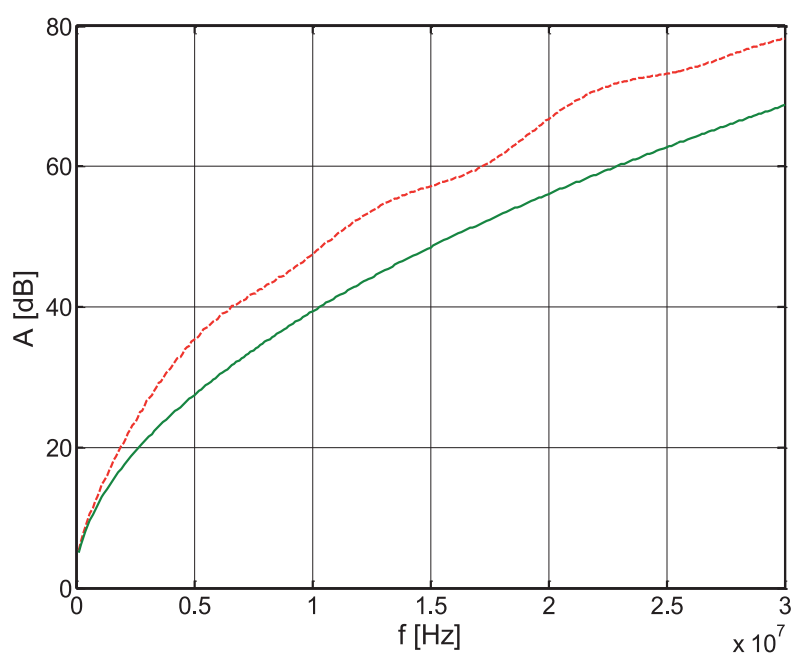

Fig. 1 Attenuation of a homogeneous line (green) and of a line with inhomogeneity and added components (red)
In order to approach the pseudo-random nature of inhomogeneity originating by cable manufacturing and installing, it is advisable to choose the lengths so that they are not integer multiples of each other. An example of modelling a line with inhomogeneity and added components (e.g. capacitance of cross-connect and distribution frames, patch cords, connection from main distribution frame to DSLAM) is shown in Figs. 1 and 2 where the value of a $500 \mathrm{~m}$ section is compared to that of a homogeneous line in a TCEPKPFLE $75 \times 4 \times 0.4$ local cable.

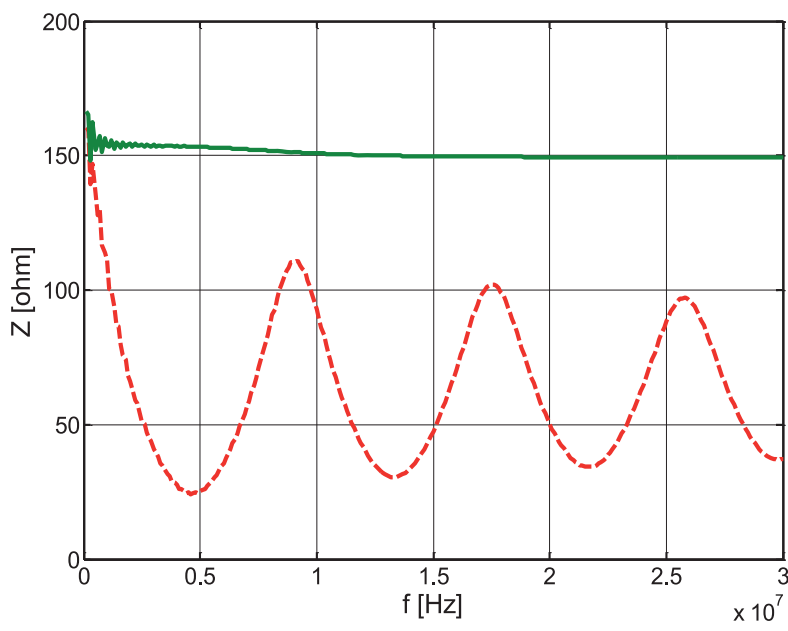

Fig. 2 Module of input impedance for a homogeneous line (green) and for a line with inhomogeneity and added components (red)

\section{Improvement code gain calculation}

Mentioned more precise calculations for line transmission parameters were implemented into Simulator xDSL program [2]. The simulation program is designed to perform calculations within the spectral compatibility sphere in a metallic access network and calculations concerning a transmission performance for several types of digital subscriber lines.

The simulation program allows performing simulation of all lines xDSL. For each transmission technology it is possible to set up an additional parameter. For example, for VDSL2 it is necessary to set up frequency band and PSD mask (Power Spectral Density). The transmission environment is modelled with the use of the noise profiles A, B, C, D, defined by ITU-T for each technology. However, it is also possible to use one's own combination of different transmission technologies.

For more precise calculations of transmission performance in Simulator XDSL program, there was a gain code correction applied. The code gain (CG) of modem depends on how the subscriber's data are protected during the transmission. What is more, a value of code gain affects bits allocation in each sub-channel of DMT 
(Discrete Multi-tone) modulation. Number of allocated bits for $\mathrm{i}$ tone (channel) is determined by (8) [5]:

$$
b_{i}=\log _{2}\left(1+\frac{S_{i}}{N_{i} \cdot k_{b}}\right)
$$

where, $b_{i}$ is the number of allocated bits, $S_{i}$ is a signal power, $N_{i}$ is a noise power. Constant $k_{b}$ is given by (9):

$$
k_{b}=\frac{S G \cdot N M}{C G}
$$

where $S G$ is Shannon Gap 9.55 for BER $=10^{-7}$, NM is Noise Margin (usually for xDSL NM $=6 \mathrm{~dB}$ ). Figure 3 shows a dependence of signal to noise ratio (SNR) on a number of channel. Blue curve is again measured, while red comes from theoretical simulation.

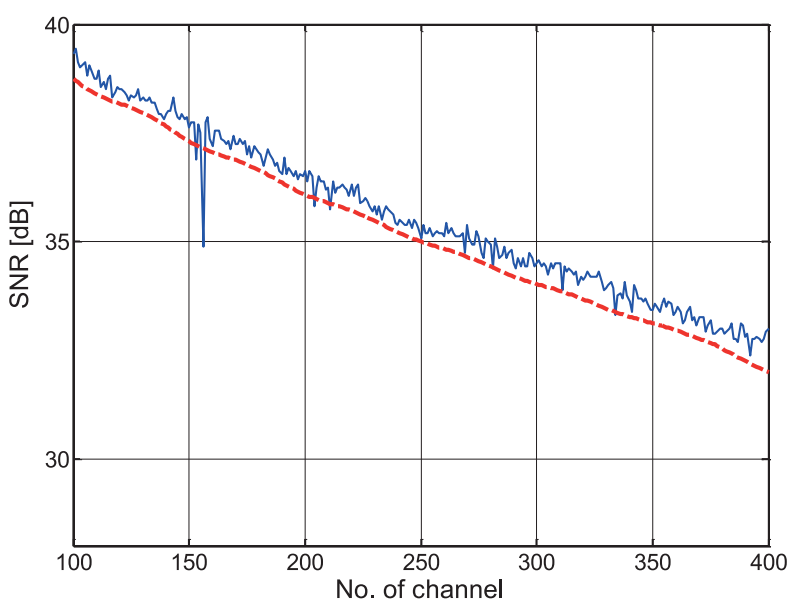

Fig. 3 Dependence of SNR on order of DMT tones

An example of code gain calculated from measured values of noise margin is shown in Fig. 4. The noise margin value varies because of the user modem that is trying to provide a maximum bitrate, which depends on the actual SNR value for a given tone. The noise margin is not really constant, but varies between the minimum and maximum values around the desired value of $6 \mathrm{~dB}$. The calculated code gain from noise margin and SNR values is around $6 \mathrm{~dB}$.

\section{Conclusion}

Two major problems were identified with precision modelling of digital subscriber lines: inhomogeneity along the line and code gain vendor dependence. The updated classification and inhomogeneity was used to refine model lines. In result, code gain value

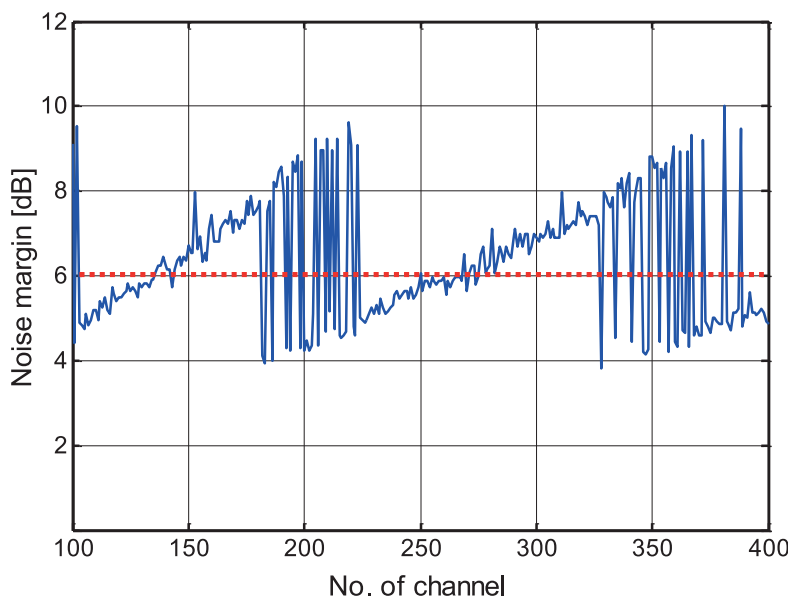

Fig. 4 Dependence of noise margin (blue) and code gain (red) on order of DMT tones

differs, depending on a vendor and algorithms implemented into the end user modem. For that reason, improvements can be applied only to a specific modem. Despite these conditions, Simulator xDSL allows to perform calculations according to specific conditions and obtain exact values for transmission performance of XDSL system. Table 2 summarizes the results before correction and after correction of the downstream bitrate modelled values for both the intended effects. Parameters of local cable TCEPKPFLE $75 \times 4 \times 0.4$, spectral profile 998ADE17 to $17 \mathrm{MHz}$ and noise model B (ETSI) were used for all cases. The noise model B is calculate from typical crosstalk from other 15 lines with VDSL2 modems and mix of SHDSL and ADSL modems in reference cable with 50 pairs.

Bitrates of VDSL2 profile 998ADE17 comparison

Table 2 for $1 \mathrm{~km}$ length of line

\begin{tabular}{|l|l|}
\hline Description & Downstream bitrate \\
\hline Effect of inhomogeneity: & $16 \mathrm{Mbps}$ \\
\hline Without inhomogeneity & $12 \mathrm{Mbps}$ \\
\hline With inhomogeneity & $12 \mathrm{Mbps}$ \\
\hline Effect of code gain correction: & $16 \mathrm{Mbps}$ \\
\hline Original value of code gain $=3 \mathrm{~dB}$ & \\
\hline New value of code gain $=6 \mathrm{~dB}$ &
\end{tabular}

\section{Acknowledgement}

This work was supported by the Grant of the Technology Agency of the Czech Republic, No. TA02011015, "Research and development of a new communication system with multi-channel approach and multi-layer co-operation for industrial applications", and was researched in cooperation with CERTICON. 


\section{References}

[1] VODRAZKA, J., JARES, P., HRAD, J.: Optimal Position of External Node for Very-high-bitrate Digital Subscriber Line, $34^{\text {th }}$ Intern. Conference on Telecommunications and Signal Processing (TSP). Budapest, AUG 18-20, 2011. Pages: 175-177. ISBN: 978-1-45771411-5

[2] JARES, P., VODRAZKA, J.: xDSL Simulator, on-line http://matlab.fel.cvut.cz/en

[3] SMIESKO, J., URAMOVA, J.: Access Node Dimensioning for IPTV Traffic Using Effective Bandwidth, Communications - Scientific Letters of the University of Zilina, No. 2, 2012. ISSN 1335-4205.

[4] LAFATA, P., VODRAZKA, J.: Simulations and Statistical Evaluations of FEXT Crosstalk in xDSL Systems Using Metallic Cable Constructional Arrangement, TSP - $31^{\text {st }}$ Intern. Conference Telecommunications and Signal Processing [CD-ROM]. Budapest: Asszisztencia Szervezo Kft., 2008, ISBN 978-963-06-5487-6.

[5] STARR, T., SORBARA, M., CIOFFI, J. M., SILVERMAN, P.: DSL Advances. Upper Saddle River, USA: Prentice Hall, 576 p., 2002. ISBN 0-13-093810-6.

[6] HRASNICA, H.: Broadband Powerline Communications Networks, John Wiley \& Sons : Chichester, 2004. ISBN 0-470-85741-2.

[7] VODRAZKA, J., HRAD, J.: Modeling of a Subscriber Line with Inhomogeneity, $32^{\text {nd }}$ Intern. Conference on Telecommunications and Signal Processing (TSP 2009), Dunakiliti, pp. 79-83, 2009. ISBN 978-963-06-7716-5. 